\title{
Asociación entre tatuajes, perforaciones y conductas de riesgo en adolescentes
}

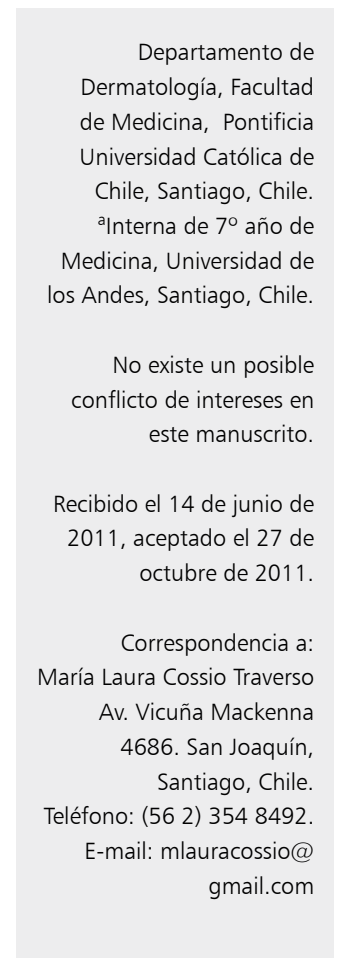

\author{
MARÍA LAURA COSSIO T., LAURA GIESEN F. ${ }^{a}$, \\ GABRIELA ARAYA, MARÍA LUISA PÉREZ-COTAPOS S.
}

\section{Association between tattoos, piercings and risk behaviors in adolescents}

Background: The use of tattoos and piercings has increased, especially among adolescents in the last decades. Aim: To evaluate the prevalence of these behaviors in adolescents and their association with risk behaviors such as alcohol, tobacco and illicit drug use and sexual promiscuity. Material and Methods: An anonymous and confidential survey about tattooing and piercings was applied to randomly selected high school teenagers, attending municipal, private-subsidized and private schools, in four sectors of Santiago (north-east, south-east, north-west, south-west). Results: The surveys were answered by 1329 participants with a mean age of 15 years (62\% women) from 9 schools in Santiago. The prevalence of tattoos was $1.7 \%$ (confidence intervals (CI) $1.1 \%$ to 2.5\%). The figure for piercings was $30.6 \%$ (CI 28.2 to $33.1 \%$ ). A higher prevalence of tattooing and piercings was observed in groups with a history of psychiatric disorders, criminal records, alcohol, tobacco and illicit drug consumption and initiation of sexual activity $(p<0,001)$. Conclusions: This study confirms that tattoos and piercings are indicators of adolescent risk behaviors.

(Rev Med Chile 2012; 140: 198-206).

Key words: Adolescence; Body piercing; Tattooing.

L a modificación del aspecto externo es una característica humana universal que comprende tanto la vestimenta, peinados, joyas y otros accesorios, así como el "arte corporal" (body art), en el que se incluyen los tatuajes y las perforaciones ${ }^{1,2}$. Estos últimos son modificaciones corporales transitorias o permanentes, cuya frecuencia ha aumentado en la sociedad occidental en las últimas décadas.

Existen múltiples definiciones de tatuajes y perforaciones. La Academia Americana de Dermatología identifica 5 tipos de tatuajes: tatuajes traumáticos (depósito indeseado de desechos); tatuajes amateur (realizados por personas no profesionales o amigos, utilizando tinta y aguja); tatuajes profesionales (incluye tatuajes culturales, realizados según la etnia a la que pertenecen, y los modernos, realizados con pistola de tatuaje); tatuajes médicos (para delimitar el sitio de radiación); y tatuajes cosméticos (delineado permanente, etc.) ${ }^{3}$.
La mayoría de los pigmentos utilizados contienen minerales, especialmente carbón, aluminio, hierro, titanio y mercurio. También se usan pigmentos biológicos como los derivados de plantas y óxidos minerales (ocre). Actualmente, existe una gran variedad de pigmentos artificiales, que contienen colorantes sintéticos y sales minerales ${ }^{1}$.

Las perforaciones o piercing se definen como la inserción de un elemento ornamental en tejidos blandos como cejas, hélix y lóbulo auricular, labios, lengua, nariz, ombligo, pezones y genitales ${ }^{1,4}$. Los elementos ornamentales son frecuentemente metálicos (níquel, plata, acero, titanio, niobio), pueden contener piedras preciosas, o pueden estar fabricados con materiales quirúrgicos y polímeros plásticos termoestables como PTFE (politetrafluoretileno), madera o hueso ${ }^{1}$.

La prevalencia de estas modificaciones corporales es variable de una población a otra, y aumenta según la edad de los sujetos estudiados. Se estima 
que 5 a $10 \%$ de los adolescentes entre 12 y 22 años declaran haberse realizado un tatuaje permanen$\mathrm{te}^{1,5,6}$. En mayores de 18 años, el porcentaje asciende a más de $20 \%$, lo que coincide con la observación de que la prevalencia de tatuajes aumenta con la edad ${ }^{7}$.

Respecto a las perforaciones, cerca de $30 \%$ de los adolescentes entre 12 y 22 años se habrían realizado alguna vez una perforación, excluyendo el lóbulo auricular en las mujeres ${ }^{10}$. En adultos, a diferencia de los tatuajes, la frecuencia disminuye con la edad, observándose una prevalencia de perforaciones entre 10 y $15 \%{ }^{7-9}$.

En Chile existen pocos datos al respecto. Un estudio realizado en el año 2002 en 934 escolares de enseñanza media de 3 comunas de Santiago mostró que cerca de $42 \%$ de los encuestados había tenido al menos una perforación ${ }^{10}$.

Tanto los tatuajes como las perforaciones tienen numerosas complicaciones médicas, las cuales han sido descritas en una revisión previa ${ }^{1}$.

Existen pocos estudios que se enfoquen en las motivaciones de quienes se realizan tatuajes o perforaciones, pero casi todos coinciden en algunos puntos, agrupándolas en las siguientes categorías $^{11,12}$.

- Belleza, arte y moda: embellecimiento.Individualidad: distinguirse de otros.

- Historia personal: catarsis personal o expresión de valores o experiencias importantes.

- Resistencia física: querer superar el propio umbral del dolor.

- Afiliación a grupos.

- Protesta: especialmente en adolescentes, contra padres, autoridades o sociedad.

- Espiritualidad y tradición cultural: pertenecer a culturas o etnias donde estas modificaciones corporales tienen significado.

- Motivaciones sexuales: perforaciones en pezones o genitales para lograr mayor placer sexual o tatuajes para expresar orientación sexual.

- Sin razones específicas: incluye amplio grupo de personas que actúan sin una razón específica y/o de manera impulsiva.

En adolescentes, la presencia de tatuajes o perforaciones se ha asociado a mayor frecuencia de abuso de alcohol y drogas ilícitas, inicio precoz de actividad sexual, mayor número de parejas sexuales, nivel socioeconómico bajo, familia uniparental, desórdenes de alimentación, violencia, problemas escolares, e ideación suicida ${ }^{5,6,13,14}$. En adultos estas asociaciones son similares, existiendo correlación entre estas modificaciones corporales y abuso de sustancias, menor nivel educacional y antecedentes penales ${ }^{7}$.

En este escenario surgen interrogantes como ¿cuál es la prevalencia de tatuajes y piercings entre los adolescentes de Santiago? ¿Es similar a la publicada en países desarrollados o nuestra realidad en Chile es distinta? ¿Hay asociación entre tatuajes y/o piercings y conductas de riesgo como las anteriormente descritas en los adolescentes de Santiago de Chile? ¿Es necesario indagar sobre estas conductas frente a cada paciente que presenta este tipo de modificaciones corporales?

En este estudio se intentará responder a estas interrogantes, con el objetivo de establecer la prevalencia de tatuajes y perforaciones en adolescentes de Enseñanza Media de Santiago, y su distribución por sexo, edad, comuna de residencia, tipo de colegio y nivel educacional de los padres, y determinar la asociación existente entre tatuajes y perforaciones, y conductas de riesgo como consumo de alcohol, tabaco, drogas ilícitas, y promiscuidad sexual.

\section{Material y Método}

Se realizó un estudio transversal (survey), con fines descriptivos (determinar frecuencia de tatuajes y perforaciones) y analíticos (determinar si existe relación entre tatuajes y perforaciones y conductas de riesgo) en adolescentes de enseñanza media de Santiago.

Se diseñó una encuesta (Anexo 1) para ser contestada por cada participante, en forma anónima y confidencial.

Se excluyeron del estudio a los participantes, padres, apoderados, o institución educacional, que rechazaran completar la encuesta en forma anónima y confidencial.

El tamaño muestral se calculó a partir de la población entre 14 y 19 años de 34 comunas de Santiago. Para esto se utilizó la prevalencia de perforaciones encontrada en un estudio piloto realizado el año 2002 , que fue de $42 \%{ }^{15}$. Se consideró un error alfa de $0,05 \%$ y error beta de $0,1 \%$, lo que arrojó un $n$ total a encuestar de 1082 participantes.

Para la selección de los sujetos a encuestar se realizó un muestreo estratificado que comprendió los siguientes pasos:

- Se dividieron las 34 comunas de Santiago en 4 sectores: 
- Nor-oriente: Las Condes, Providencia, Vitacura, Lo Barnechea y La Reina.

- Sur-oriente: Macul, Nuñoa, Peñalolén, La Florida y Puente Alto.

- Nor-poniente: Conchalí, Huechuraba, Independencia, Recoleta, Quilicura, Renca, Santiago Centro, Cerro Navia, Estación Central, Lo Prado, Pudahuel y Quinta Normal.

- Sur-poniente: Cerrillos, Lo Espejo, Maipú, Pedro Aguirre Cerda, El Bosque, La Cisterna, La Granja, La Pintana, San Bernardo, San Joaquín, San Miguel y San Ramón.

- Se seleccionó una muestra proporcional a la población entre 14 y 19 años, y al número de alumnos matriculados en colegios municipalizados, particulares-subvencionados y particulares, para cada uno de los cuatro sectores.

- Los colegios presentes en cada sector, se eligieron en forma aleatoria.

A cada uno de los colegios seleccionados, se envió una carta explicando los objetivos del proyecto. Posteriormente, se entregó un formulario de consentimiento informado para ser firmado por las autoridades y/o profesores del colegio, quienes informaron a los padres y apoderados sobre el proyecto.

En cada colegio, previo a la aplicación de la Encuesta, se realizó una presentación en "Power Point", con el fin de informar a los alumnos y profesores sobre los riesgos y complicaciones que conllevan la realización de tatuajes y perforaciones. A continuación se contestaron las dudas de los alumnos, y posteriormente se aplicó la encuesta, en forma anónima y confidencial. Este proceso fue realizado por la misma investigadora en todos los colegios, durante julio y octubre de 2008.

Los datos obtenidos fueron tabulados en una planilla Excel.

Se realizaron tablas de frecuencia de las distintas variables estudiadas, tanto en la muestra total como en subgrupos y tablas de contingencia donde se aplicó la prueba de $\chi^{2}$. Para la comparación de medias se utilizó la prueba t de Student.

\section{Resultados}

\section{Características de la Muestra}

Se obtuvo 1.329 encuestas válidamente contestadas. Se aplicó el cuestionario a estudiantes de $1^{\circ} \mathrm{a}$ $4^{\circ}$ medio ( 14 a 19 años) de 9 colegios de los cuatro sectores de Santiago, de los cuales 3 fueron colegios Municipales, 4 colegios Particular-Subvencionado y 2 Particulares. La distribución por sexo fue $62 \%$ hombres y $38 \%$ mujeres. La edad promedio fue 15 años, encontrándose el $95 \%$ de la muestra entre los 14 y 16 años. La distribución de la muestra en los cuatro sectores de Santiago según comuna de residencia fue la siguiente: $17 \%$ Nor-Oriente; $31,5 \%$ Nor-Poniente; $24,8 \%$ Sur-Oriente y $17,2 \%$ Sur-Poniente.

Respecto a las conductas de riesgo estudiadas, $42 \%$ refirió consumir alcohol, de los cuales un tercio lo hacía más de 1 vez por semana. El consumo de tabaco en la muestra estudiada alcanzó $25,4 \%$. La frecuencia de consumo de drogas ilícitas observada fue de $8,4 \%$, correspondiendo el $92 \%$ a marihuana. El 23,6\% de los encuestados afirmó haber tenido alguna vez actividad sexual, de los cuales $21 \%$ refería haber tenido más de 3 parejas en los últimos 12 meses.

La prevalencia de tatuajes observada fue $1,7 \%$ (IC 1,1\% a 2,5\%); mientras que la de perforaciones fue $30,6 \%$ (IC 28,2 a $33,1 \%$ ). Al analizar la prevalencia en los 4 sectores de Santiago se encontró que ésta fue significativamente mayor tanto para tatuajes como piercing en el sector Sur-Oriente, comparado con el sector Nor-Oriente $(3,3 \%$ vs $0,5 \%$ en tatuajes, con $\mathrm{p}=0,01 ;$ y $39,5 \%$ vs $28,3 \%$ en perforaciones, con $\mathrm{p}=0,03$ ) (Figuras 1 y 2).

No se encontró diferencias significativas en la prevalencia de tatuajes según sexo, tipo de colegio, nivel educacional de ambos padres y realización de actividad deportiva regular. La prevalencia de tatuajes sí fue mayor en grupos con antecedente de patología psiquiátrica, antecedentes penales, consumo de alcohol, tabaco y drogas ilícitas y con inicio de actividad sexual $(\mathrm{p}<0,001)$. También se encontró que, quienes afirmaban pertenecer a la religión católica tenían menor prevalencia de tatuajes que aquellos que no pertenecían a ninguna religión $(\mathrm{p}=0,002)$.

La prevalencia de perforaciones, en cambio, fue mayor en colegios municipales que en particulares-subvencionados, y ésta mayor que en colegios particulares, observándose una asociación lineal $(\mathrm{p}=0,04)$ (Figura 3$)$.

El sexo femenino mostró una prevalencia de piercing significativamente mayor que el sexo masculino $(43,1 \%$ vs $22,9 \%, \mathrm{p}<0,001)$. La actividad deportiva frecuente se asoció a una menor prevalencia de perforaciones $(\mathrm{p}=0,03)$; mientras 


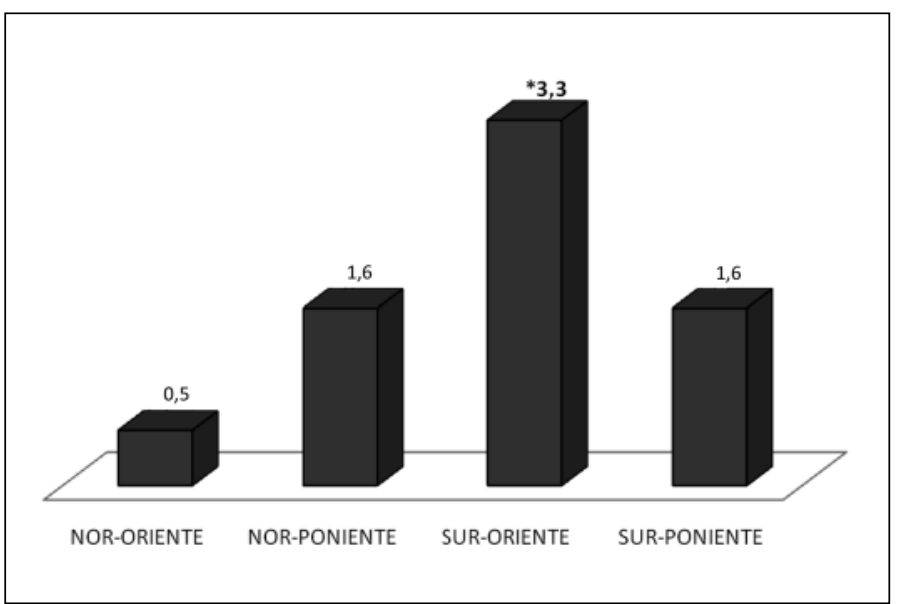

Figura 1. Prevalencia tatuajes por sector.

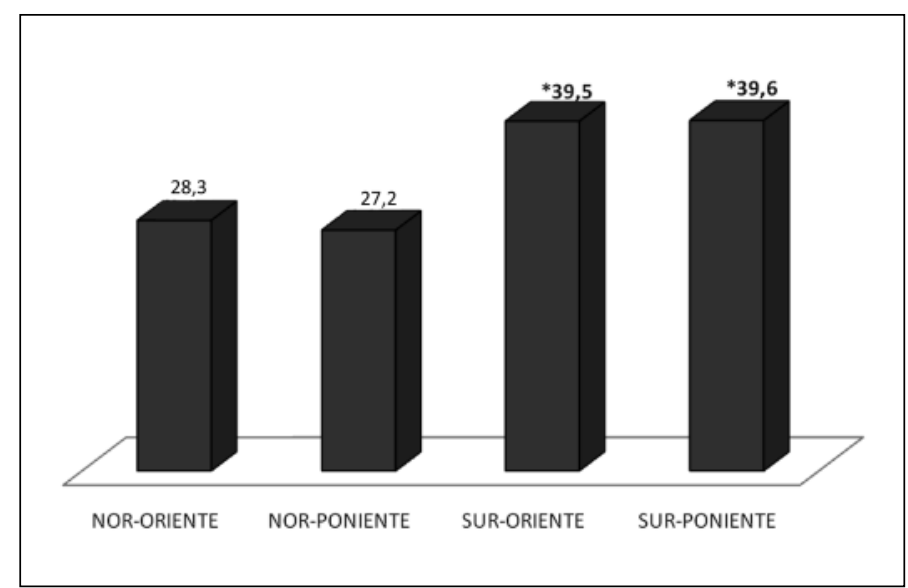

Figura 2. Prevalencia perforaciones por sector.

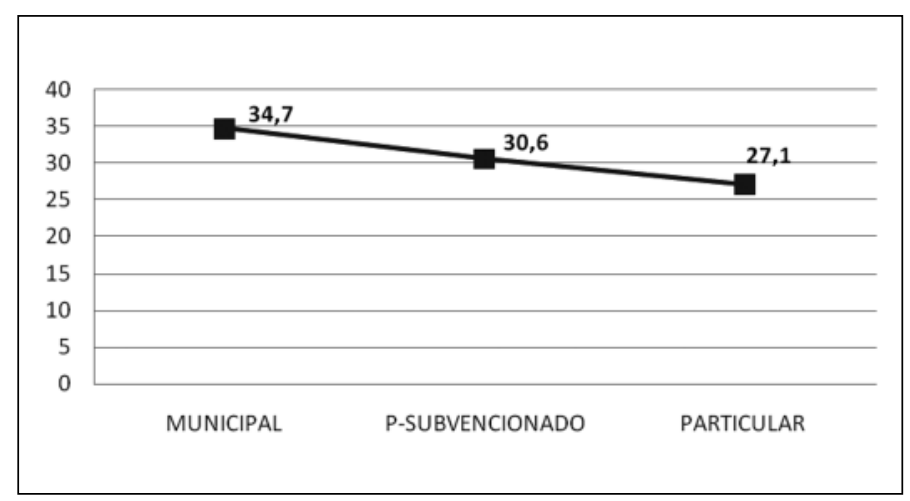

Figura 3. Prevalencia perforaciones por tipo colegio. que el antecedente de patología psiquiátrica, la presencia de antecedentes penales, el consumo de tabaco, alcohol y drogas ilícitas, y el inicio de actividad sexual, se asociaron significativamente a mayor prevalencia de perforaciones $(p<0,001)$.

La edad promedio en los sujetos con tatuajes fue 15,45 años, la que es significativamente mayor que la de aquellos sin tatuajes $(p=0,01)$. También se encontró que los individuos con tatuajes consumían alcohol con mayor frecuencia, tenían mayor número de perforaciones, y la edad a la que adquirían el primer piercing fue menor que la de aquellos sin tatuajes $(\mathrm{p}<0,05)$.

En los sujetos con perforaciones se observó mayor frecuencia de consumo de alcohol $(\mathrm{p}<0,01)$.

Tanto para tatuajes como para perforaciones, no se encontraron diferencias estadísticamente significativas en cuanto a la localización de estos por área de la piel entre hombre y mujeres. Las zonas más frecuentemente tatuadas fueron en primer lugar la espalda y en segundo lugar los brazos (Figura 4); mientras que las perforaciones fueron más prevalentes en orejas (exceptuando el lóbulo auricular en mujeres, que fue excluido del estudio), cara (incluyendo cejas, nariz y labios) y lengua (Figura 5).

La aparición de complicaciones, tanto para tatuajes como para piercing, no tuvo relación con la localización de éstos por área corporal ni el lugar donde se adquirieron. Sin embargo, llama la atención que $44 \%$ de los tatuajes y $63 \%$ de los piercing, los hayan adquirido en forma amateur (en feria artesanal o por un amigo). Respecto a las complicaciones, sólo uno de los sujetos del grupo con tatuajes reportó una infección local. De los individuos con perforaciones, en cambio, $18,4 \%$ refirió haber tenido alguna complicación, de las cuales el $89,3 \%$ fueron infecciones locales. 


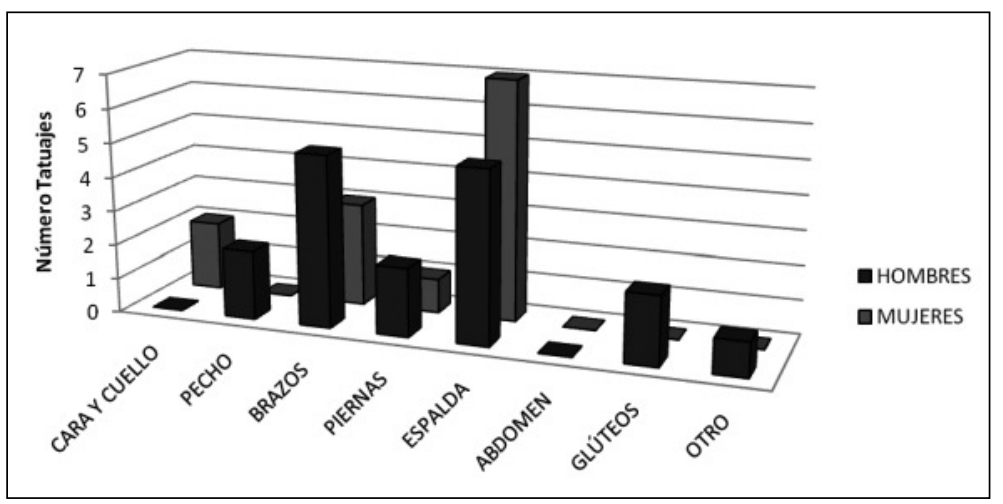

Figura 4. Localización tatuajes por sexo.

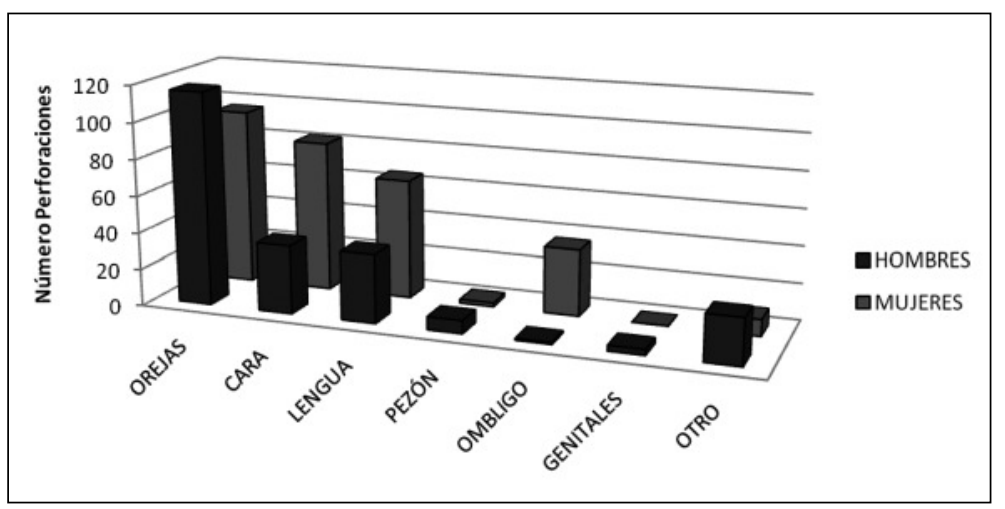

Figura 5. Localización perforaciones por sexo.

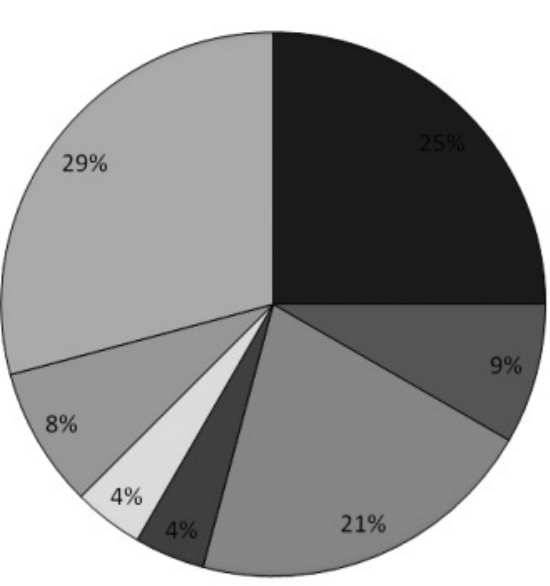

- ESTÉTICA (MODA-ARTE)

$\square$ RESISTENCIA AL DOLOR

- PERTENENCIA A GRUPO

口INDIVIDUALIDAD

口EXPERIENCIA PERSONAL

口INESPECÍFICO

口PROTESTA

Figura 6. Motivaciones para realizarse tatuajes.

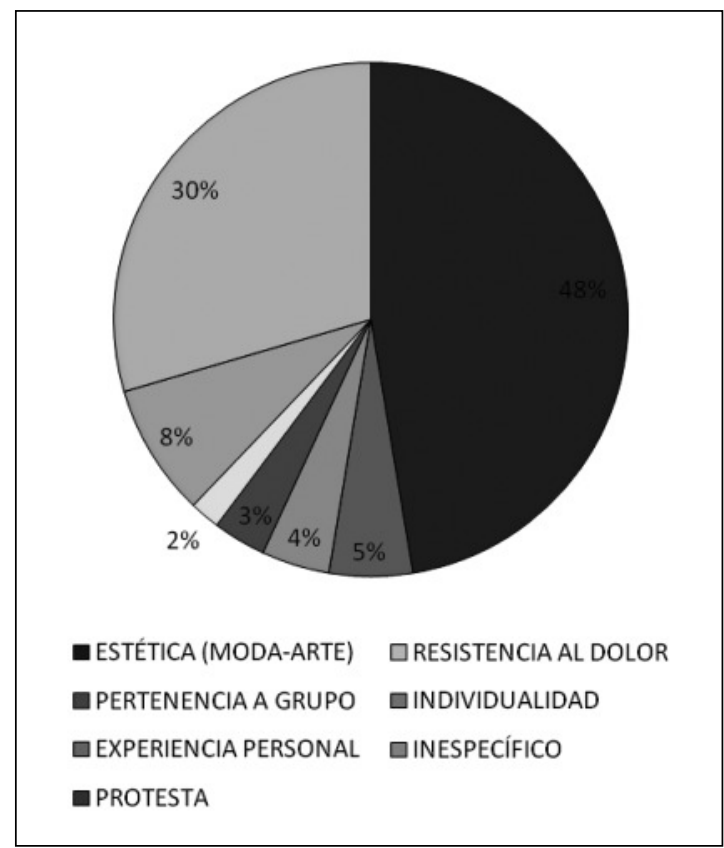

Figura 7. Motivaciones para realizarse perforaciones. 
Las motivaciones para realizarse un tatuaje o perforación no se asociaron a las otras variables estudiadas. En ambos casos las más frecuentes fueron las motivaciones estéticas (moda y arte) y su adquisición como un acto impulsivo, sin motivación específica (Figuras 6 y 7 ).

\section{Discusión}

La prevalencia de perforaciones (30\%) observada en este estudio, coincide con lo reportado en la literatura internacional, mientras que la prevalencia de tatuajes $(1,7 \%)$, fue significativamente inferior (cercana al 5\%). Esto podría tener relación con la edad de los encuestados, ya que, según lo reportado en estudios extranjeros, la frecuencia de tatuajes aumenta a mayor edad ${ }^{7}$, y quizá en Chile la adquisición de estos sea más tardía que en otros países. A esto se suma que en la distribución por edades de la muestra, se observa un predominio de edades menores (14 a 16 años), lo que podría influir en la menor prevalencia de tatuajes encontrada.

La mayor prevalencia de tatuajes y perforaciones observada en el sector Sur-Oriente, comparado con el Nor-Oriente puede sugerir que en comunas con predominio de nivel socioeconómico medio y medio-bajo serían más frecuentes este tipo de modificaciones. Sin embargo, este dato debe interpretarse con cautela ya que la distribución de la muestra por sectores no fue homogénea en cuanto a sexo ni edad. En este mismo sentido, la mayor prevalencia de perforaciones observada en colegios municipales y particulares-subvencionados que en colegios particulares, podría ser un indicador de que el nivel socioeconómico juega algún rol.

La mayor prevalencia de perforaciones en mujeres, a pesar de que la muestra es predominantemente masculina, podría explicarse por una mayor aceptación social de estas prácticas en el sexo femenino, además de motivaciones estéticas.

La correlación entre tatuajes, perforaciones y conductas de riesgo en adolescentes, si bien está descrita en la literatura, no había sido estudiada en Chile, y muchos pensaban que el aumento de estas prácticas tenía más relación con una moda entre jóvenes que con la presencia de dichos comportamientos. Este estudio, a pesar de ser un estudio piloto, confirma que existe asociación entre patología psiquiátrica, antecedentes penales, consumo de alcohol, tabaco y drogas ilícitas, e inicio de actividad sexual, y mayor prevalencia de tatuajes y perforaciones entre los adolescentes de Enseñanza Media de Santiago.

Un hallazgo interesante, es la actividad deportiva regular asociada a una menor prevalencia de perforaciones, lo que podría relacionarse con hábitos de vida más saludables.

Otra variable que no había sido estudiada en adolescentes, aunque sí en adultos, es la religión. En esta muestra, pertenecer a la religión católica se asocia a menor prevalencia de tatuajes al compararlo con aquellos que no pertenecen a ninguna religión.

También se encontró que la presencia de tatuajes se asocia a mayor número de perforaciones, y a una edad inferior para adquirir el primer piercing, comparado con la población sin tatuajes.

A pesar de no encontrarse asociación entre el lugar de adquisición del tatuaje o perforación, y la aparición de complicaciones médicas, llama la atención que la mayoría refiere haberse realizado estas modificaciones en forma amateur (en feria artesanal o con un amigo), lo que sin duda, debe ser objeto de futuras investigaciones, además de un tema de educación y discusión entre adolescentes, profesores y apoderados.

Una limitación de este estudio es que si bien la distribución de los sujetos por cada sector y tipo de colegio es proporcional a la población objetivo, existe un sesgo de selección determinado por la participación voluntaria, por lo que es posible que la frecuencia de tatuajes y perforaciones en dichos colegios fuese inferior a la encontrada en el estudio. A su vez, la distribución de la muestra por edad no tiene la misma distribución que la población general de enseñanza media ya que, en la mayoría de los colegios se realizó a alumnos de primero y segundo medio (14 a 16 años).

\section{Conclusión}

Como estudio piloto, este proyecto estima que la prevalencia de perforaciones entre adolescentes de Santiago sería similar a la reportada en estudios extranjeros. A su vez confirma que la presencia de tatuajes y perforaciones son indicador de conductas de riesgo en adolescentes, abriendo paso a otro tipo de investigaciones que aborden, por ejemplo, el perfil psicológico de los jóvenes que tienen este 
tipo de conductas, su realidad familiar y escolar, y su posible relación con patologías psiquiátricas. Además sugiere tanto a padres, educadores como a los propios médicos, que deben estar alerta ante este tipo de modificaciones corporales.

\section{Referencias}

1. Pérez-Cotapos ML, Cossio ML. Tatuajes y perforaciones en adolescentes. Rev Méd Chile 2006; 134: 1310-7.

2. Nicoletti A. Teens, tattoos and body piercing. J Pediatr Adolesc Gynecol 2004; 17: 215-6.

3. Goldstein N. Tattoos defined. Clin Dermatol 2007; 25: 417-20.

4. Stirn A. Body piercing: medical consequences and psychological motivations. Lancet 2003; 361: 1205-15.

5. Roberts T, Ryan S. Tattooing and high-risk behavior in adolescents. Pediatrics 2002; 110: 1058-63.

6. Carroll S, Riffenburgh R, Roberts T, Myhre E. Tattoos and body piercings as indicators of adolescent risktaking behaviors. Pediatrics 2002; 109: 1021-7.

7. Laumann A, Derick J. Tattoos and body piercings in the United States: a national data set. J Am Acad Dermatol 2006; 55: 413-21.
8. Bone A, Ncube F, Nichols T, Noah ND. Body piercing in England: a survey of piercing at sites other than earlobe. BMJ 2008; 336: 1426-8.

9. Gutsche P, Schmalz G, Landthaler M. Prevalence of piercing in a German population. Eur J Dermatol 2008; 18: 26-8.

10. Fuenzalida $\mathrm{H}$, Álvarez E, Martin C. Piercing en un grupo de población escolar santiaguina. Rev Chil Dermatol 2003; 19: 271-6.

11. Wohlrab S, Stahl J, Kappeler P. Modifying the body: motivations for getting tattooed and pierced. Body Image 2007; 4: 87-95.

12. Tiggemann M, Golder F. Tattooing: an expression of uniqueness in the appearance domain. Body Image 2006; 3 : 309-15.

13. Stephens MB. Behavioral risks associated with tattooing. Fam Med 2003; 35: 52-4.

14. Koch JR, Roberts AE, Armstrong ML, Owen DC. Frequencies and relations of body piercing and sexual experience in college students. Psychol Rep 2007; 101: 159-62.

15. Chimenos E, Batlle I, Velásquez S, García T, Viñals H, Roselló X. Appearance and culture: oral pathology associated with certain "fashions". Med Oral 2003; 8: 197-206.

Anexo 1. Cuestionario de salud: factores epidemiológicos asociados al uso de tatuajes y piercings

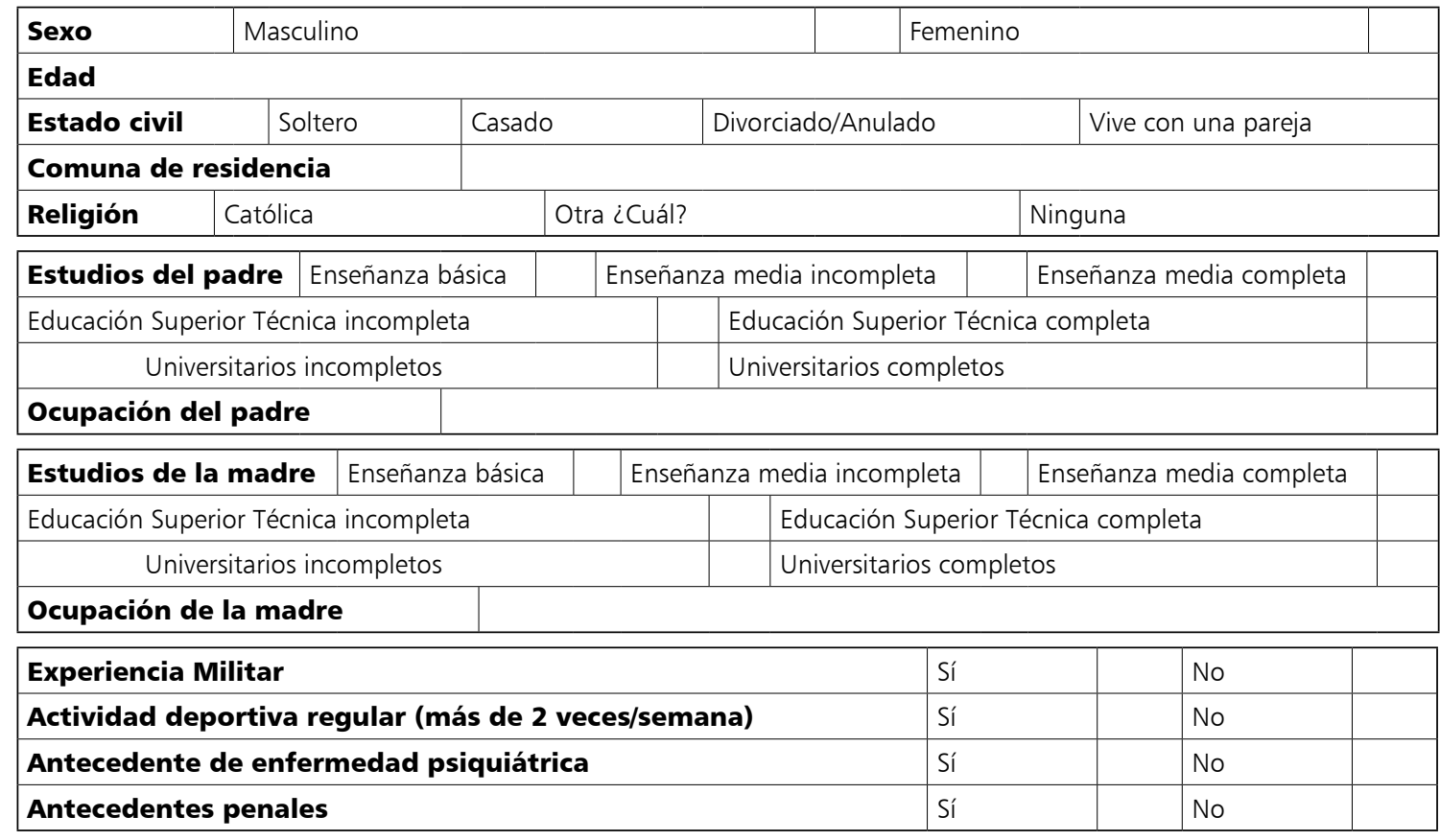


Asociación entre tatuajes y perforaciones con conductas de riesgo - M. L. Cossio T. et al

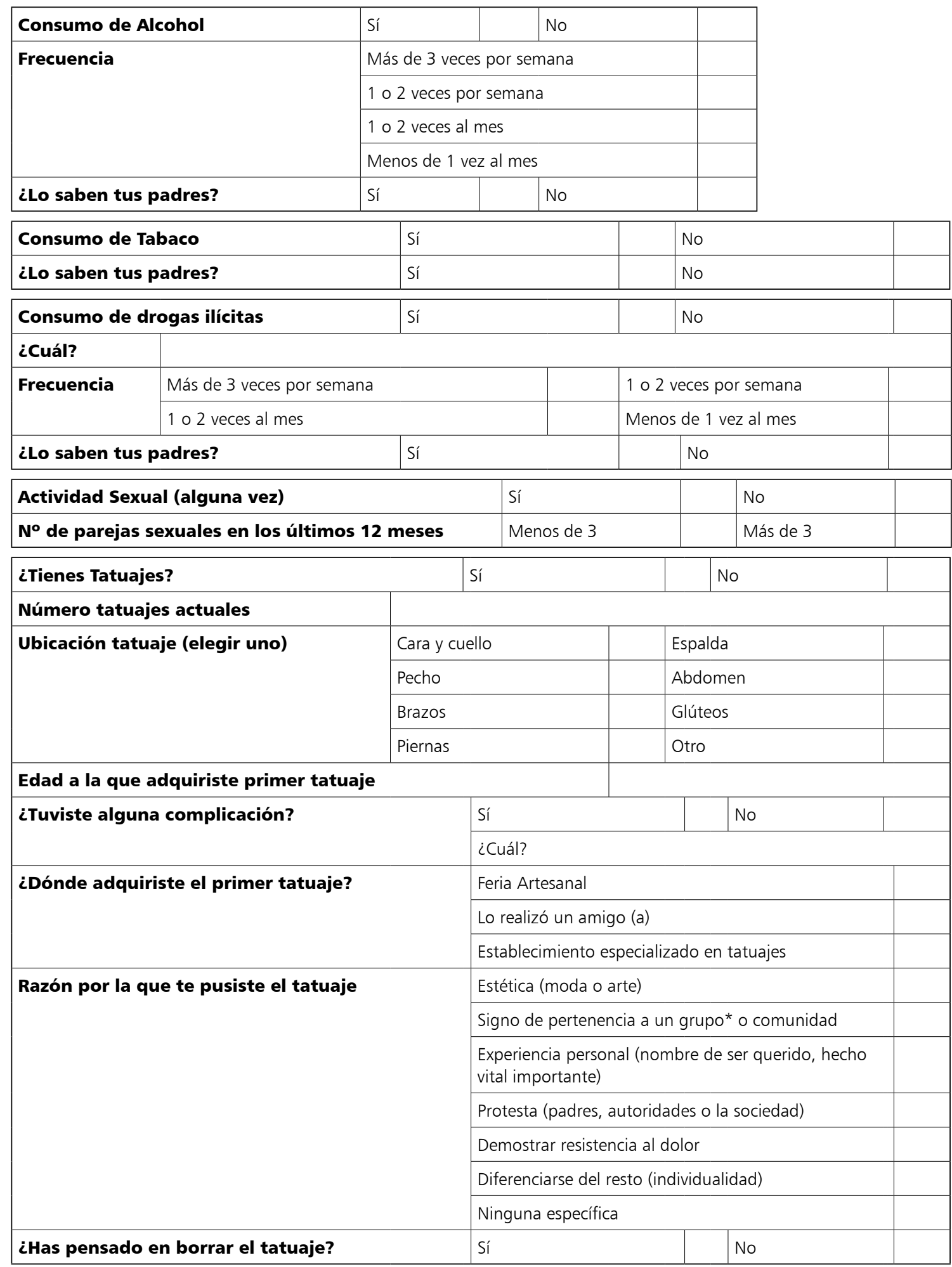

*Equipo deportivo, banda de música, grupo de amigos, tribus, etc. 


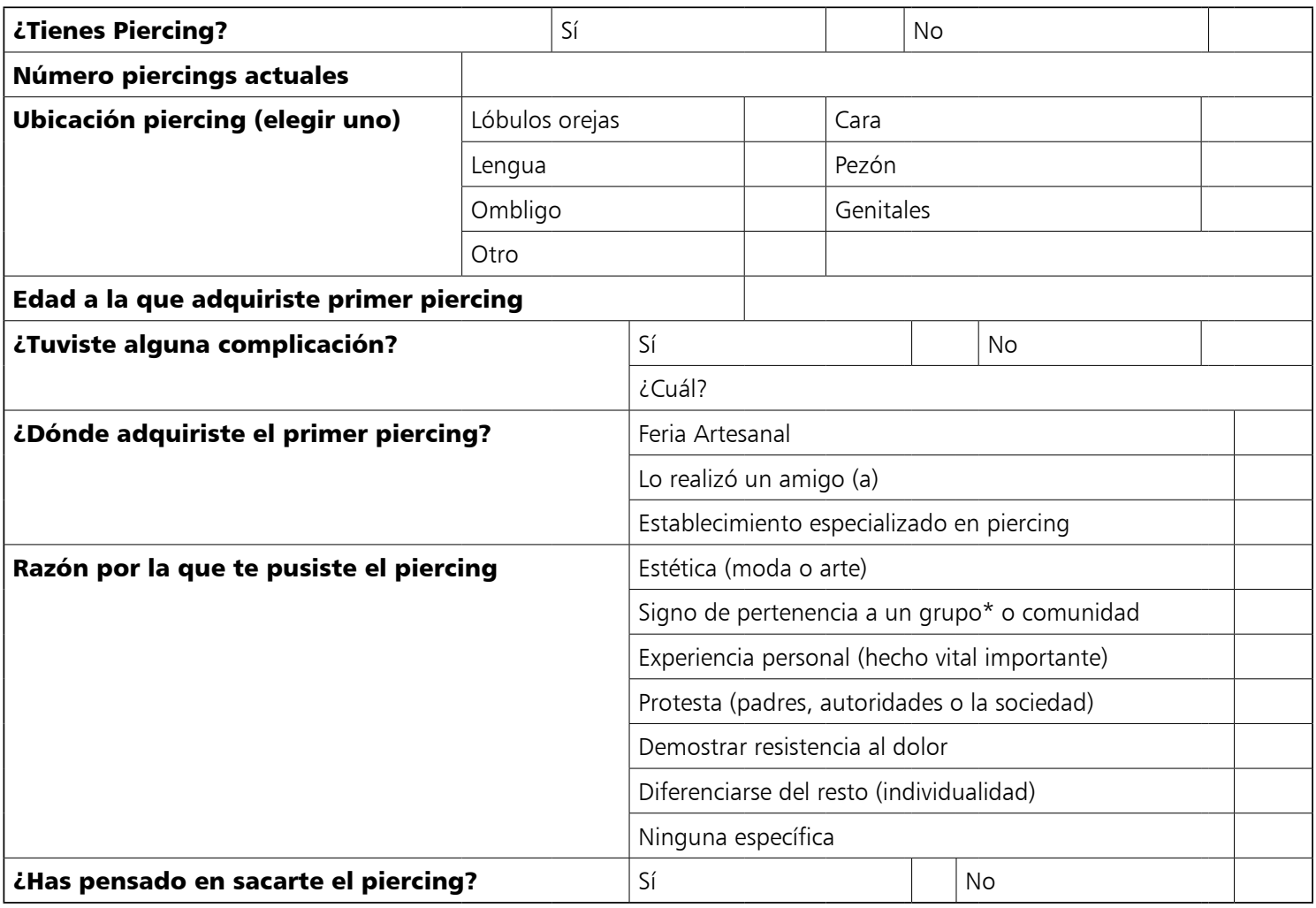

*Equipo deportivo, banda de música, grupo de amigos, tribus, etc. 